\title{
Canadians' Views on Palliative Care
}

\author{
Erin Roulston, MA
}

\begin{abstract}
Palliative Care Matters is a national initiative designed to develop consensus on the steps that need to be taken now to ensure Canadians can access high-quality palliative care services as part of Canada's universal healthcare model. Covenant Health and the Canadian Partnership Against Cancer joined with leading national health organizations to lead this initiative, resulting in a Consensus Development Conference in November 2016. Six questions were identified by experts in the field to form the basis of the conference. The questions were first explored with Canadians through focus groups to assess views toward the topics. The qualitative research results were then used to develop an online survey conducted with 1540 Canadians to gauge views on palliative care. The survey results identified what essential elements should be included in a palliative care (and home care) program. Canadians express a desire for palliative healthcare providers to have "specialized certifications" and they have a moderate level of self-confidence in their own palliative caregiving knowledge and abilities. Awareness of palliative care is not widespread in Canada, but support for a modest educational public health campaign is prevalent. Canadians also strongly support national palliative care standards and integration into the Canada Health Act, but simultaneously express financial concerns regarding the associated costs required. The public opinion survey results were used as part of the deliberations at the Consensus Development Conference, culminating in a consensus statement being issued to inform potential policy options and implementation plans for palliative care in Canada.
\end{abstract}

Keywords: focus groups; palliative care; palliative caregiving; palliative care standards; palliative healthcare providers; public health campaign; survey; universal healthcare model

$\boldsymbol{P}$ alliative CaRe Matters is a national initiative designed to talk with Canadians about their views and lived experiences, review the most current evidence, and develop consensus on the steps that need to be taken now to ensure Canadians in all parts of the country can access high-quality palliative care services as part of Canada's universal healthcare model. Covenant Health has joined with many of Canada's leading national health organizations and experts in palliative care and health policy to lead this initiative, culminating in a national Consensus Development Conference.

Six questions were identified to form the basis of the conference. The questions were determined by experts in the field and include the most important areas needing to be addressed.

The Canadian Reference Working Group, comprised of a variety of experts in the palliative care field, identified the following six questions that were presented by the Expert Panel and posed to the Lay Panel during the conference:
1. What are the essential elements of an integrated and coordinated palliative care program that will help improve access to quality palliative care in Canada?

2. Do public health awareness campaigns effectively improve the awareness and quality of palliative care?

3. What are the essential components of quality palliative home care services for all Canadians?

4. What resources are required to ensure adequate education, training, and mentorship for all healthcare providers and caregivers of Canadians experiencing a life-limiting illness and their families?

5. What have strategic frameworks and plans accomplished for palliative care when adopted by countries?

6. Does measurement of indicators that address desired outcomes, including patient-/family-reported outcomes and experience, improve the quality of and access to palliative care? 
Before the conference, Palliative Care Matters sought public opinion related to the six questions and engaged Ipsos to conduct the research. Palliative Care Matters acknowledges the Canadian Partnership Against Cancer for their considerable contributions to funding this research.

\section{Research Methodology}

The public opinion research involved two phases:

\section{Phase 1}

Qualitative exploratory research: Before measuring public opinion related to the six conference questions, a series of focus groups was conducted with Canadians (including a good mix of demographics and one homogeneous focus group with Indigenous representatives) in Edmonton, Toronto, and Montreal to identify views on these topics. The groups were held between June 28th and July 4th, 2016 and the feedback informed the design of the subsequent survey questions and response categories.

\section{Phase 2}

Quantitative survey research: Armed with information gleaned from the initial qualitative research, Ipsos worked with the Palliative Care Matters team to develop a survey questionnaire aimed to measure Canadians' views related to the six conference topics. The online survey was conducted among 1540 Canadians aged 18 years and older between August 2nd and 11th, 2016. The survey sample was stratified among regions within Canada and quotas were placed to align with Statistics Canada census data by region, age, and gender. While margins of error are not reported for online surveys, had this survey been conducted with a probability sample, the overall margin of error would be $\pm 2.5 \%, 19$ times out of 20 .

Results of the national survey were presented by Ipsos at the Consensus Development Conference, which was held November 7-9, 2016 in Ottawa. At the conference, the Lay Panel studied what Canadians said and heard from experts on the key issues that need to be addressed to improve palliative care.
Following their deliberations, the Lay Panel issued a consensus statement detailing their findings and proposing next steps.

\section{Summary of Public Opinion Research Findings}

Results of the public opinion research are categorized by the six conference topics, as follows:

Question 1: What are the essential elements of an integrated and coordinated palliative care program that will help to improve access to quality palliative care in Canada?

Expectations for program elements are very high and need to be carefully managed.

After being presented with a series of 27 potential elements for a palliative care program in Canada, most Canadians agree that all 27 elements should be included, denoting high expectations should a palliative care program be developed.

The most essential elements involve pain management (92\%) and personal hygiene (92\%). In addition, nursing care specialized in palliative care $(91 \%)$, medications $(91 \%)$, and meals $(91 \%)$ is also deemed highly important, followed by having a physician specialized in palliative care $(90 \%)$ and having someone in the system to coordinate palliative care services (88\%), among others. Elements that rank lower, more as "nice-to-have" versus "need-to-have," involve pet therapy $(64 \%)$, faith leaders $(65 \%)$, translation services $(66 \%)$, music therapy (67\%), and access to Wi-Fi (67\%) (Fig. 1).

Canadians have a healthy appetite for additional and higher quality healthcare services, with Canadians ranking healthcare as the 2nd most important issue facing Canada. It will likely be challenging to include all desired elements of a palliative care program in Canada within the current economic conditions in the country (Canadians identify economic issues at the top of the Canadian issues agenda).

In context, satisfaction with palliative care currently appears to be quite positive. Of the $51 \%$ of Canadians who have had someone important to them receive palliative care within the past 10 years, $89 \%$ are satisfied. However, when looking

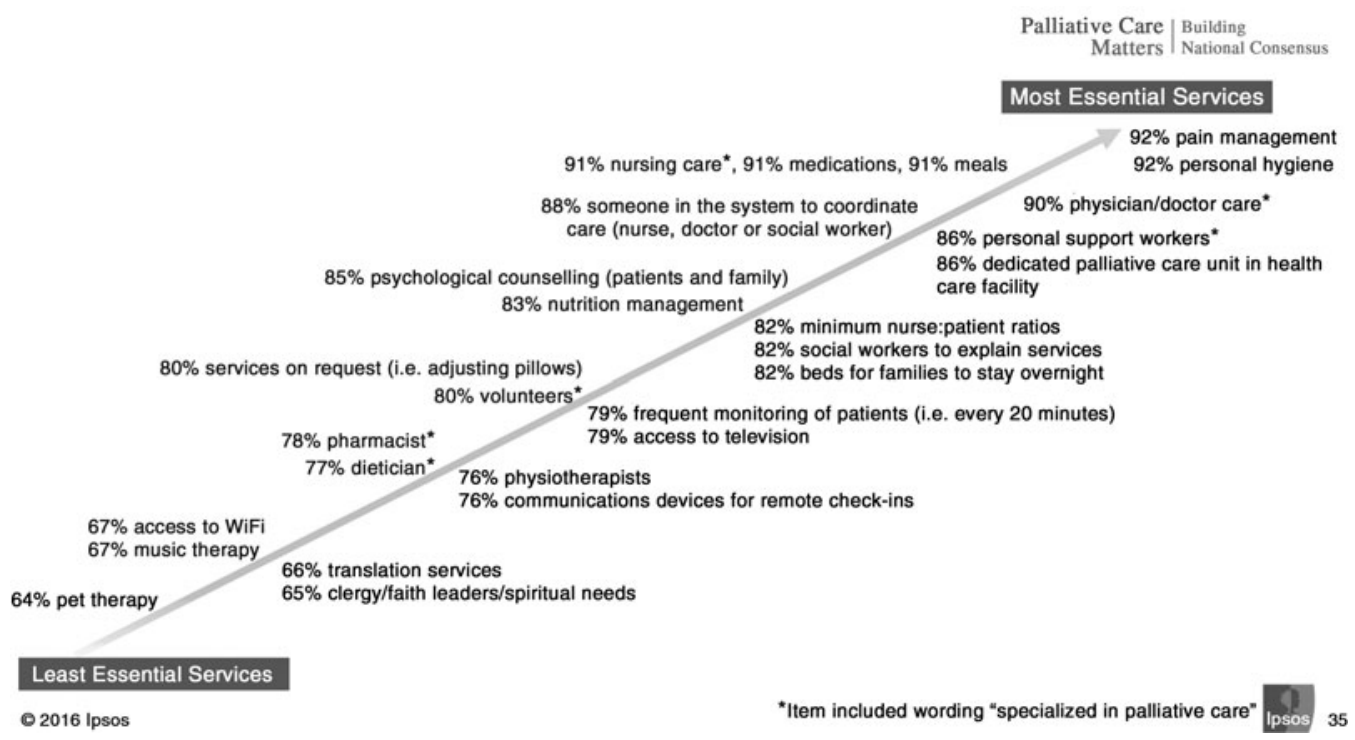

FIG. 1. Essential elements of a palliative care program. 


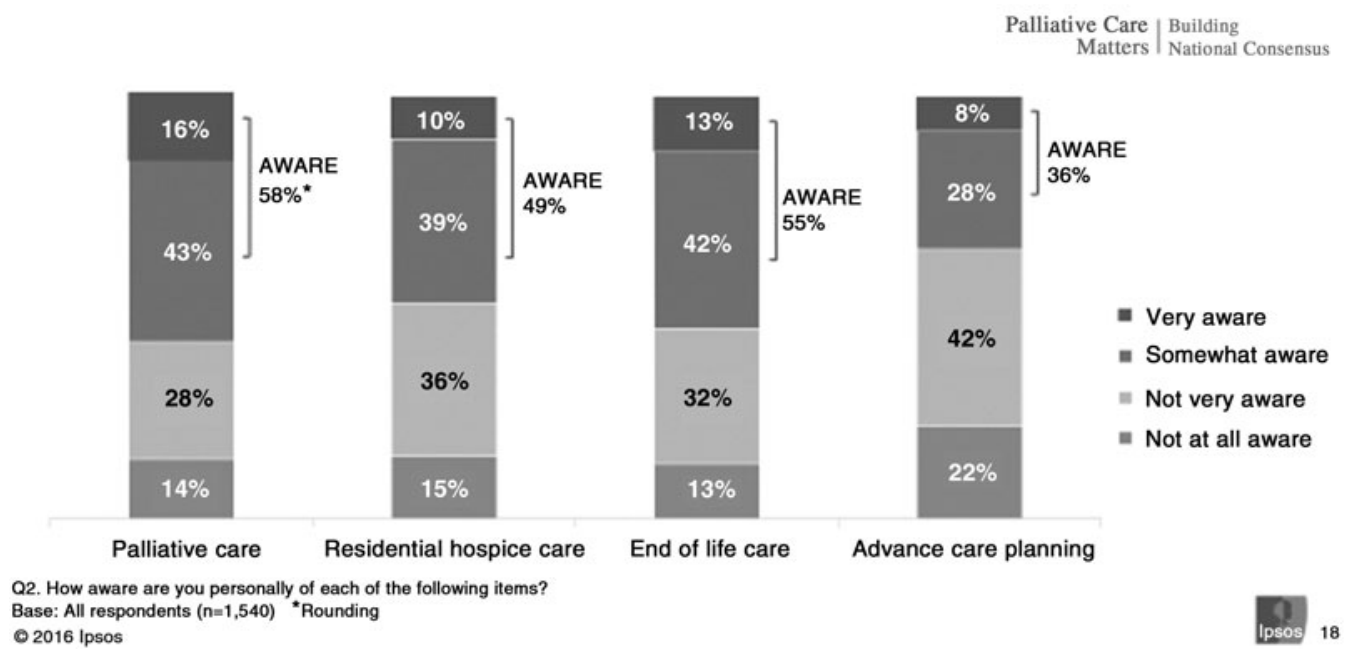

FIG. 2. Awareness of palliative care.

at satisfaction among those currently providing care, lower satisfaction ratings emerge (78\%), which may indicate a decline in satisfaction recently and/or be impacted by current caregivers' state of mind in a difficult time.

Question 2: Do public awareness campaigns effectively improve the awareness and quality of palliative care?

Awareness of palliative care is not widespread in Canada, but support for a "reasonably priced" public health campaign is.

Awareness of palliative care is found among $58 \%$ of Canadians, including just $16 \%$ who say they are "very aware" of what this entails (Fig. 2). Therefore, there are many more Canadians to educate about palliative care. They are primarily getting their information about palliative care through word of mouth (family, friends, and news), and the government and medical community do not "own" the content of the messages, which may or may not be correct.

To ensure that Canadians know that quality palliative healthcare is available, $85 \%$ of Canadians agree that a public awareness campaign should be conducted to inform Canadians about palliative healthcare services and standards, including $46 \%$ who "strongly agree" and 39\% who "somewhat agree."

In another measure, $42 \%$ of Canadians feel that a public health campaign regarding palliative care standards should "definitely" be undertaken, and an additional $41 \%$ believe that such a campaign should be undertaken "only if it entails reasonable costs" (Fig. 3). How "reasonable" is defined by Canadians is unknown, but it appears that strong support for a modest campaign exists.

To properly answer Conference Question \#2, a campaign would have to be implemented and at the end of the "media buy," awareness and satisfaction could be measured again (as well as measuring exposure to the campaign) and compared against the benchmarks captured in this study. When researchers see statistically significant increases in awareness and satisfaction and analyze correlations to campaign exposure, the question can be more appropriately answered.

In the meantime, the benchmark data suggest that there is not only an opportunity but also support for a public health campaign to make a difference in both awareness and the quality of palliative care, especially if done in tandem with

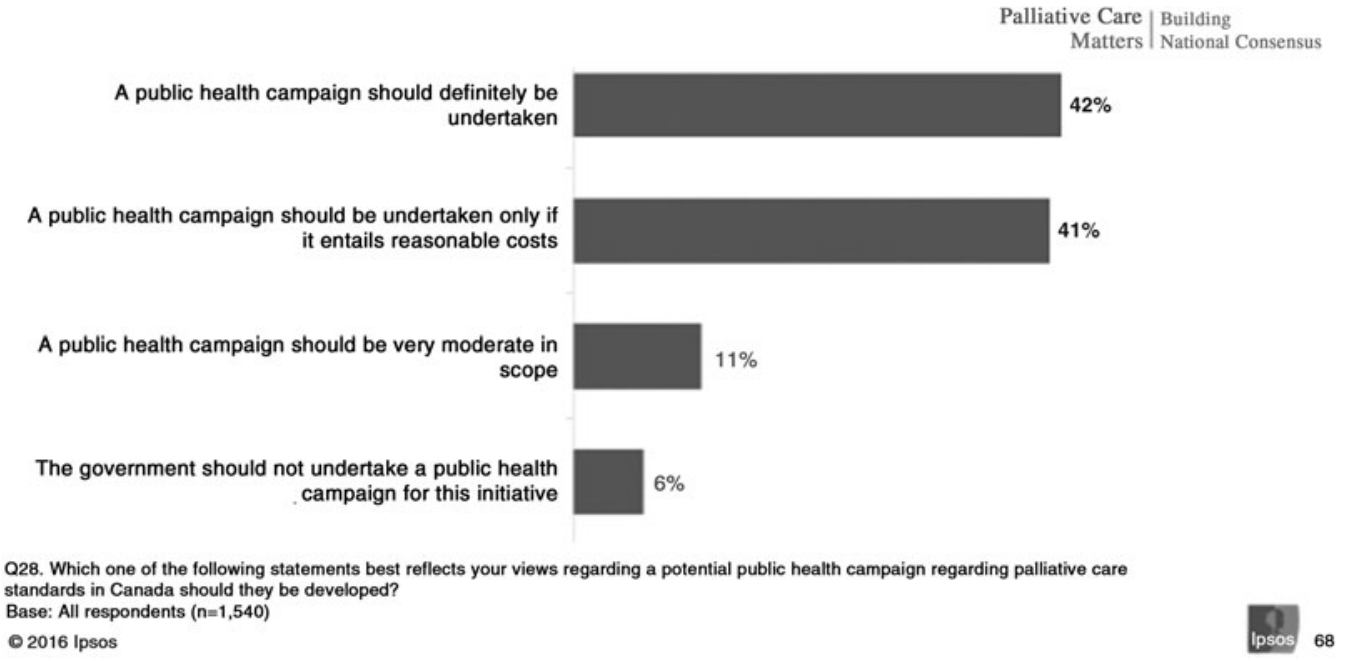

FIG. 3. Views regarding public health campaigns. 


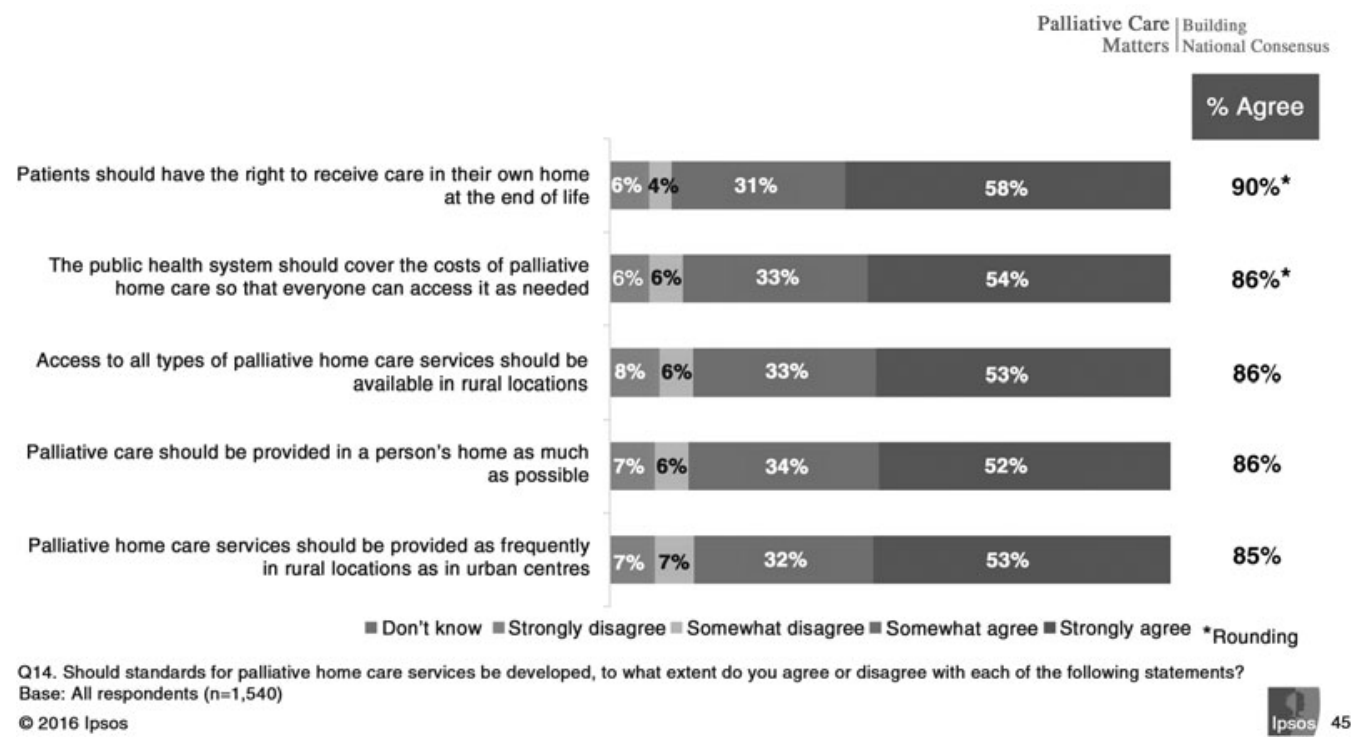

FIG. 4. Attitudes toward palliative home care.

other activities to ensure adequate education and training of palliative care providers and caregivers.

Question 3: What are the essential components of quality palliative home care services for all Canadians?

Expectations for a palliative home care program are also very high.

In addition to being presented with a list of 27 elements, which could be included in a national palliative healthcare program in a healthcare facility, survey respondents were presented with a list of 15 additional elements that could apply to a palliative home care program.

A strong majority of Canadians agree that all 15 elements of a potential palliative home care program should be included. The strongest agreement is found for having 24/7 access to a nurse $(91 \%)$, daily assistance with caregiving $(91 \%)$, access to equipment as needed $(91 \%)$, panic alerts/ buttons for patients when left alone (91\%), education and information for caregivers (91\%), someone in the system to coordinate care $(91 \%)$, and a single access point (i.e., one number to call) for help $(90 \%)$. The lowest agreement expressed (although still strong at $80 \%$ ) is found for renovation funding (i.e., bathroom bars, ramps, and vehicles).

Furthermore, a robust majority of Canadians agree that patients should have the right to receive care in their home at the end of life (90\%) and the public health system should cover any associated costs (86\%). In addition, Canadians agree that palliative home care should be provided in rural locations $(86 \%)$, in a person's home as much as possible $(86 \%)$, and as frequently in rural locations as in urban centers (85\%) (Fig. 4).

Canadians see value in a palliative home care program, but the integration of all of the desired elements will have to be explored to understand if all palliative home care components are logistically and financially feasible.

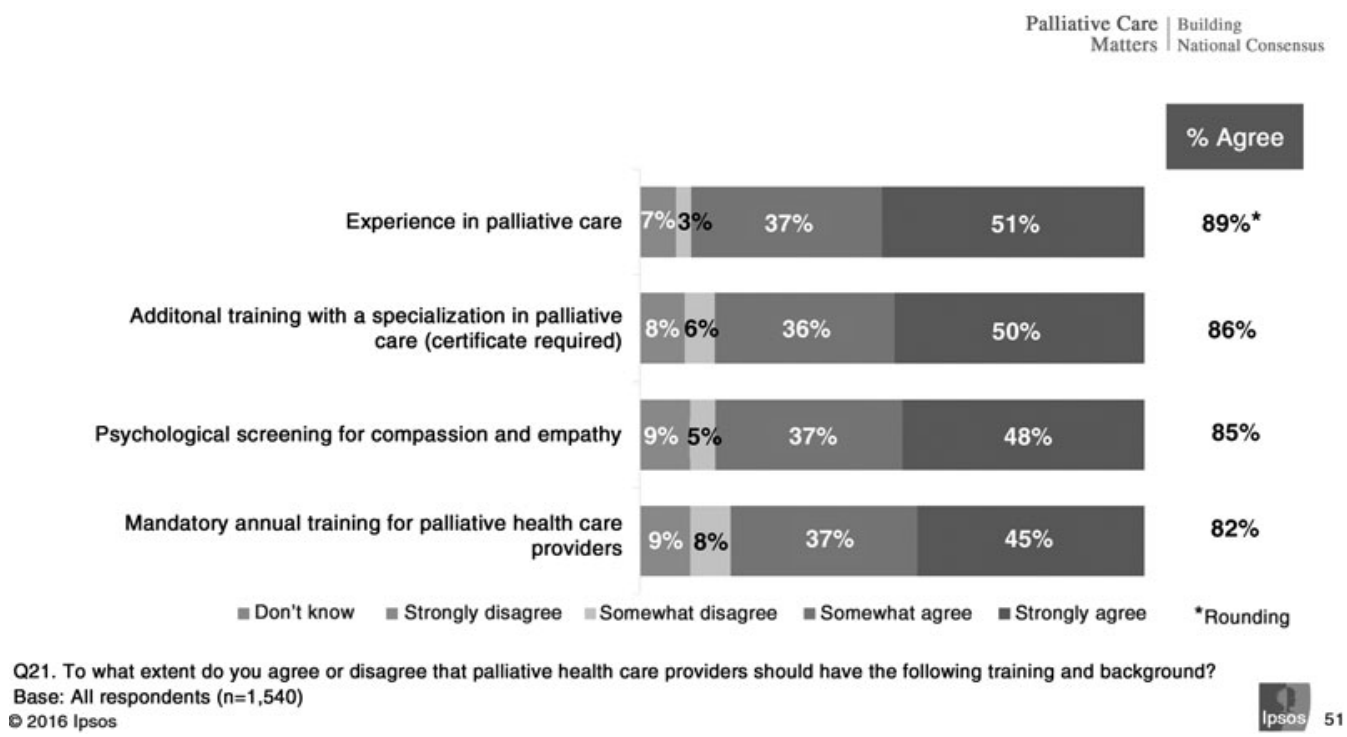

FIG. 5. Mandatory qualifications for healthcare providers. 


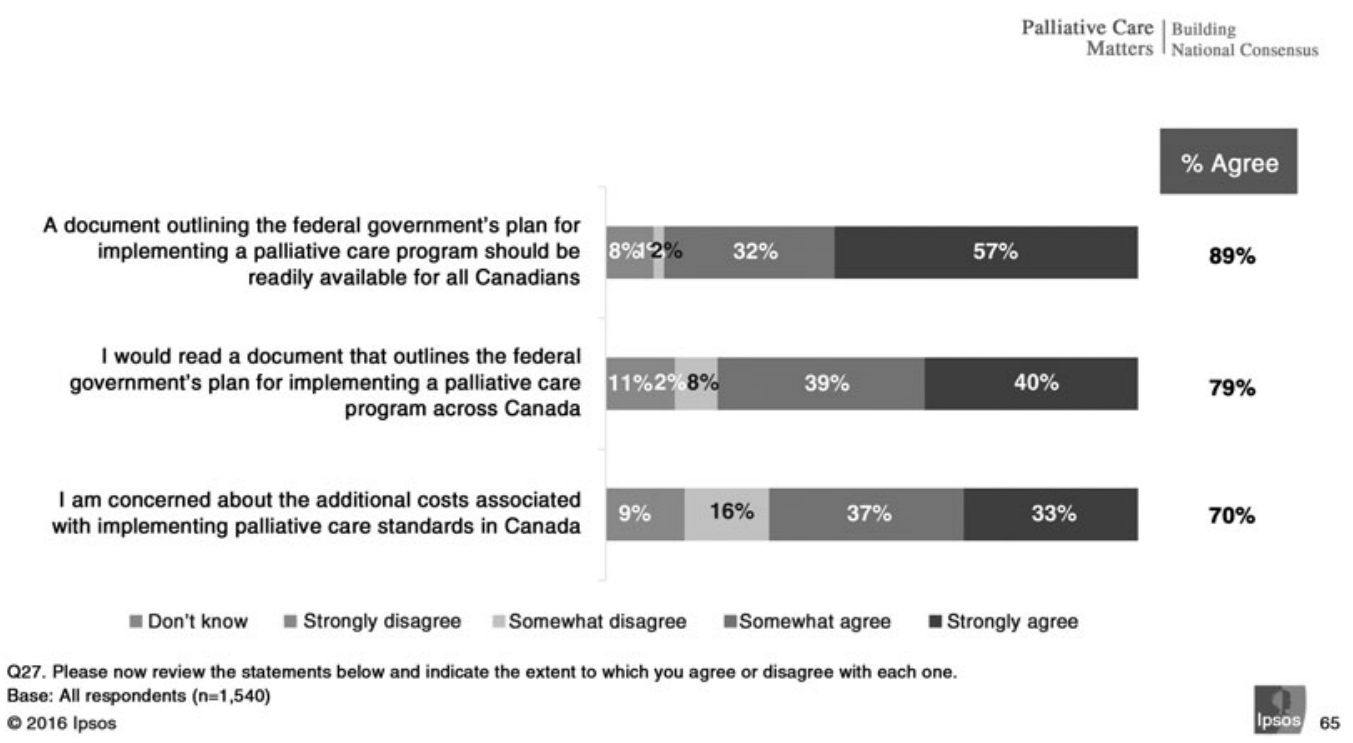

FIG. 6. Attitudes: implementing a palliative care program.

Question 4: What resources are required to ensure adequate education, training, and mentorship for all healthcare providers and caregivers of Canadians experiencing a life-limiting illness and their families?

Confidence in palliative caregiving is moderate and Canadians prefer to be trained by those within the health system.

Almost one-quarter (24\%) of Canadians have been (20\%) or are currently (4\%) caregiving for someone important to them who was or is dealing with a life-threatening illness, and among this subgroup, $77 \%$ are confident in providing the necessary care. If Canadians were caring for a close friend or family member who was dealing with a life-threatening illness, priority information needs involve who to call in an emergency $(73 \%)$, how to use machines $(72 \%)$, what to do when a patient dies at home $(72 \%)$, how to give medication $(71 \%)$, and information about dedicated care providers
(70\%). Canadians would also seek information about options for financial support $(68 \%)$, the patient's diet $(67 \%)$, and what supplies are needed (64\%), among other topics.

Canadians feel that hospitals or healthcare facilities in which the palliative care is being provided (46\%) should be responsible for providing information and training for caregivers. Just more than three-in-ten Canadians place such responsibility on family doctors $(32 \%)$ and nurses $(31 \%)$, followed by provincial (29\%) and federal (21\%) governments. Canadians desire "specialized certifications" for palliative healthcare providers, along with psychological screening.

With respect to palliative healthcare providers' training and background, a sizeable majority of Canadians feel that they should have direct experience in palliative care (89\%). Also, $86 \%$ agree that palliative healthcare providers should receive certification for additional training specialized in palliative care. Canadians also largely agree $(85 \%)$ that psychological
Not coordinated with other necessary services. Small rural community. Not much assistance. Mostly done with family and friends with the insistence of the patient. No continuity with other caregivers which was unsettling to the patient.

The staff not well trained or are too busy handling too many patients at the same time.

I think the care was the best they could offer at the time. However, I felt resources were spread thin and someone wasn't always available to my family member when they were needed.

The people who provide the care are inconsistent, too many poorly trained individuals providing the care, mistakes are quickly covered up, care is extremely expensive.

Insufficient resources available to ensure proper care.
The staff was not available to provide care when requested. Answers we received from staff were incorrect as if we were in their way.

There was some difficulty/challenges with communication to family members, and issues with consistency of the team providing palliative care.

The doctors kept the patient so drugged up that they had no quality of life. When my sister-in-law went into the hospice she was coherent and able to get around. Within 24 hours she was strapped into a diaper and so doped up she was unable to talk to her family. It was easier for the staff to care for her that way. Her meals were left by her bedside but because she was always unconscious she very seldom ate. If family members were in to visit, we would feed her, but the staff did nothing for her at all. It was a cruel way to spend the last few weeks of her life.

Q6. And why do you say that you are dissatisfied with the palliative care that the person important to you received? Base: Those who had someone important to them receive palliative care $(n=x x x)$

- 2016 Ipsos

FIG. 7. Opportunities for improvement: verbatims. 
screening for compassion and empathy should be part of palliative healthcare providers' background, and $82 \%$ feel that mandatory annual training for palliative healthcare providers should be implemented (Fig. 5).

Question 5: What have strategic frameworks and plans accomplished for palliative care when adopted by countries?

While the public opinion survey cannot fully respond to the Conference's Question \#5, the public opinion research aimed to provide some insight into potential strategic frameworks for discussion.

Support for national palliative care standards and integration into the Canada Health Act are strong, but financial concerns exist.

A sizeable majority (86\%) of Canadians expect the federal government to develop and implement national standards for palliative care in Canada, and $85 \%$ support integrating national standards for palliative healthcare services into the Canada Health Act.

Nonetheless, $70 \%$ of Canadians are concerned about the additional costs associated with implementing palliative care standards in Canada. In an era of "economic issues", dominating the Canadian issues agenda, financial concerns are prevalent. A cost-benefit analysis would have to be undertaken to better understand the financial realities involved.

Financial concerns were also spontaneously brought up in the initial exploratory focus group workshops, with some participants noting that there are trade-offs for Canadians to consider, should additional taxpayer dollars be involved with the development and implementation of national standards for palliative care in Canada. To this end, $89 \%$ of Canadians would want a document outlining the federal government's plan for implementing a palliative care program to be readily available for all Canadians before proceeding (Fig. 6).

Question 6: Does measurement of indicators that address desired outcomes, including patient and family-reported outcomes and experiences, improve the quality of and access to palliative care?
Measurement of indicators that address desired outcomes can identify opportunities for improvement; only in addressing the opportunities for improvement can the quality of palliative care be improved

The majority of Canadians agree that surveys should be conducted among family members $(84 \%)$, caregivers $(82 \%)$, and patients (79\%) regarding the care received, and $72 \%$ of Canadians agree that annual surveys should be conducted to measure and track Canadians' awareness levels of palliative care. Survey measurements can be combined into one tool for efficiencies.

Survey results can point to opportunities for improvement. For example, this survey measured satisfaction and dissatisfaction with palliative care received. Among those who had someone important to them receive palliative care, a followup open-ended question was posed to ask why they were satisfied or dissatisfied. Responses reveal that certain Canadians feel that some palliative care staff could potentially benefit from additional training, the system could use additional funds for more resources, some facilities could provide better accommodations, and communication between patients, families, and palliative healthcare providers could also be improved (Fig. 7). As these items contribute to satisfaction with the quality of palliative care, addressing these concerns from those who had poor experiences is an opportunity to improve palliative care for everyone needing such services.

Tracking measurement of indicators over time will provide an assessment of improvements in the quality of palliative care.

Address correspondence to: Erin Roulston, MA Ipsos

Canada Public Affairs

700 6th Avenue SW, Suite 1950 Calgary, Alberta T2P OT8

Canada

E-mail: erin.roulston@ipsos.com 